\title{
Expression of the Mouse Na-K-2CI Cotransporter, mBSC2, in the Terminal Inner Medullary Collecting Duct, the Glomerular and Extraglomerular Mesangium, and the Glomerular Afferent Arteriole
}

\author{
Mark R. Kaplan, ${ }^{*}$ Matthew D. Plotkin, ${ }^{*}$ Dennis Brown, ${ }^{\ddagger}$ Steven C. Hebert, ${ }^{\star}$ and Eric Delpire* \\ $*$ Renal Division, Department of Medicine, Brigham and Women’s Hospital and Harvard Medical School; and ${ }^{\ddagger}$ Renal Unit, Department of \\ Pathology, Massachusetts General Hospital and Harvard Medical School, Boston, Massachusetts 02115
}

\begin{abstract}
$\mathrm{Na}-\mathrm{K}-\mathrm{Cl}$ cotransport plays an important role in the kidney in $\mathrm{NaCl}$ reabsorption in the thick ascending limb of Henle and a less well defined role in the inner medullary collecting duct (IMCD). Two Na-K-Cl cotransporters encoded by different genes have been identified in the mammalian kidney: BSC1/NKCC2 which localizes to the apical thick ascending limb of Henle and BSC2/NKCC1 which was isolated from a mouse IMCD cell line (mIMCD-3) but its localization has not been determined. In this study we generated a polyclonal antibody (anti-mBSC2) against the mouse BSC2/ NKCC1 protein in order to characterize and localize this protein in mouse kidney. Western blot analysis with affinity-purified anti-mBSC2 showed a protein doublet of 140 and $150 \mathrm{kD}$ which was most abundant in the renal papilla but also seen in cortex and outer medulla. The 140-150-kD bands were not seen with preimmune serum or with antimBSC2 preabsorbed with specific antigen. Immunolocalization confirmed expression of $\mathrm{mBSC} 2$ protein on the basolateral surface of terminal IMCD segments and demonstrated expression in the papillary surface epithelium. Immunofluorescence also revealed the unexpected presence of the BSC2 protein at the juxtaglomerular afferent arteriole, in a juxtaglomerular structure probably representing the extraglomerular mesangium, and throughout the glomerular mesangium. (J. Clin. Invest. 1996. 98:723-730.) Key words: tubuloglomerular feedback - renin • inner medullary collecting duct $\bullet$ mesangium $\bullet$ macula densa
\end{abstract}

\section{Introduction}

Recent molecular studies have demonstrated the existence of two distinct genes encoding the mammalian Na-K-2Cl cotransporter (for reviews see references 1 and 2). The first gene, $B S C 1$ or $N K C C 2$, located on mouse chromosome 2 (3), encodes a kidney specific Na-K-2Cl cotransporter $(4,5)$ ex-

This work was presented in part in abstract form at Experimental Biology 96, Washington D.C., April 14-17 1996.

Address correspondence to Dr. Eric Delpire, Critical Care Research Laboratories, Department of Anesthesia, Children's Hospital, 320 Longwood Avenue, Boston, MA 02115. Phone: 617-355-6526; FAX: 617-730-0435; E-mail: delpire@al.tch.harvard.edu

Received for publication 30 November 1995 and accepted in revised form 29 May 1996.

J. Clin. Invest.

(C) The American Society for Clinical Investigation, Inc.

0021-9738/96/08/0723/08 \$2.00

Volume 98, Number 3, August 1996, 723-730 pressed on the apical membrane of the thick ascending limb of Henle (TAL) ${ }^{1}$ cells (6). The other gene, BSC2 or NKCC1, located on mouse chromosome 18 (7), encodes a widely expressed Na-K-2Cl cotransporter. The BSC2 cotransporter has been isolated from the shark rectal gland (8), a human colonic cell line (9), and a mouse inner medullary collecting duct cell line (mIMCD-3) (7). Extensive transport studies in the shark rectal gland and in the T84 colonic cell line have demonstrated the presence of the cotransporter on the basolateral membrane of these epithelial cells. Transport studies in the mIMCD-3 cells also revealed a putative basolateral localization of the cotransporter (7).

The salt and water reabsorptive function of the apical, BSC1, isoform of the cotransporter in the thick ascending limb is well established based on the large natriuretic effect of loop diuretics. The salt and water secretory function of the basolateral, BSC2, isoform is also well established in secretory epithelia of respiratory tract, salivary gland, and sweat glands (10). In contrast, the role and localization of the basolateral isoform of the cotransporter in the kidney is still unknown. Precise localization of this isoform in the mammalian kidney by immunostaining techniques is therefore of primary importance in determining its role in kidney function. For this report, we prepared and purified an antibody directed against a 74-amino acid peptide located in the carboxyl-terminal tail of the mBSC2 protein. The antibody was characterized by Western blot analysis and used for localization by indirect immunofluorescence. Anti-mBSC2 antibodies revealed the following locations of the mBSC2 protein: $(a)$ the basolateral membrane of terminal IMCDs, consistent with the high level of expression in the mIMCD-3 cell line; $(b)$ the papillary surface epithelium which separates the papillary interstitium from the renal pelvis; $(c)$ the unexpected presence in a subpopulation of vascular smooth muscle cells located exclusively at the afferent arteriole in close proximity to the glomerulus; and $(d)$ a juxtaglomerular structure that probably represents the extraglomerular mesangium and to a lesser extent throughout the glomerular mesangium.

\section{Methods}

Antibody production. A fusion protein consisting of a 74-amino acid segment of the carboxyl terminus of mBSC2 (amino acids 938-1011) fused to the maltose-binding protein (MBP) was generated according to the manufacturer's instructions (New England Biolabs Inc., Beverly, MA). The mBSC2-MBP fusion protein was purified with an amylose resin column and injected subcutaneously into rabbits to

1. Abbreviations used in this paper: IMCD, inner medullary collecting duct; MBP, maltose-binding protein; TAL, thick ascending limb of Henle; TGF, tubuloglomerular feedback. 
generate polyclonal antibodies according to Antisera System protocol (HRP, Inc., Denver, PA). Preimmune and immune sera were harvested and used in the present studies.

Affinity purification of polyclonal antibodies. Cyanogen bromide coupled to Sepharose beads (Pharmacia Biotech Inc., Piscataway, $\mathrm{NJ}$ ) was activated and linked either to the fusion protein or to the MBP. Immune serum was incubated for $1-3 \mathrm{~d}$ at $4^{\circ} \mathrm{C}$ with cyanogen bromide Sepharose beads coupled to fusion protein, and specific antibodies eluted from beads with $0.1 \mathrm{M}$ glycine ( $\mathrm{pH}$ 2.7). Cyanogen bromide Sepharose beads coupled to MBP were used for negative selection of antibodies to the MBP. Purified antibody was stored in PBS containing $0.02 \%$ sodium azide at $-20^{\circ} \mathrm{C}$.

Western blots. Kidney membrane protein fractions were extracted from male CD-1 mice (Charles River Laboratories, Wilmington, MA). Briefly, dissected kidney sections were homogenized in 1-2 $\mathrm{ml}$ of homogenization medium $(0.32 \mathrm{M}$ sucrose, $5 \mathrm{mM}$ Tris hydrochloride [pH 7.5], and $2 \mathrm{mM}$ EDTA and protease inhibitors: $100 \mu \mathrm{M}$ PMSF, $2 \mu \mathrm{g} / \mathrm{ml}$ leupeptin, and $2 \mu \mathrm{g} / \mathrm{ml}$ aprotinin), and centrifuged at $3,000 \mathrm{~g}$ for $10 \mathrm{~min}$. The supernatant was centrifuged at $100,000 \mathrm{~g}$ for $30 \mathrm{~min}$. The final pellet was resuspended in 50-400 $\mu \mathrm{l}$ (depending on pellet size) of suspension buffer (5 mM Tris- $\mathrm{HCl}$ [pH 7.5] and $2 \mathrm{mM}$ EDTA) and stored at $-80^{\circ} \mathrm{C}$. Protein homogenates were resolved by Laemmli SDS-polyacrylamide (6-10\%) gel electrophoresis and transferred in $25 \mathrm{mM}$ Tris- $\mathrm{HCl}, 192 \mathrm{mM}$ glycine, $25 \%$ methanol to a polyvinylidene difluoride (PVDF) membrane. Prestained high range SDS-PAGE standards were used (Bio Rad Laboratories, Hercules, CA). The membrane was blocked for $1 \mathrm{~h}$ in $5 \%$ milk powder/TBST (10 mM Tris-HCl, pH 8.5, $150 \mathrm{mM} \mathrm{NaCl}, 0.1 \%$ Tween 20). The membrane was exposed to immune serum or purified antibody diluted in $5 \%$ milk powder/TBST overnight at $4{ }^{\circ} \mathrm{C}$ and then to the second antibody (peroxidase-linked anti-rabbit Ig from Amersham Life Science, Arlington Heights, IL) for $1 \mathrm{~h}$ at room temperature. After washing in TBST, antigen-antibody complexes were detected by autoradiography using chemiluminescence (ECL, Western blotting analysis system from Amersham Life Science). For immunoabsorption studies, fusion protein was preincubated with purified antibody at room temperature for $1-3 \mathrm{~h}$.
A

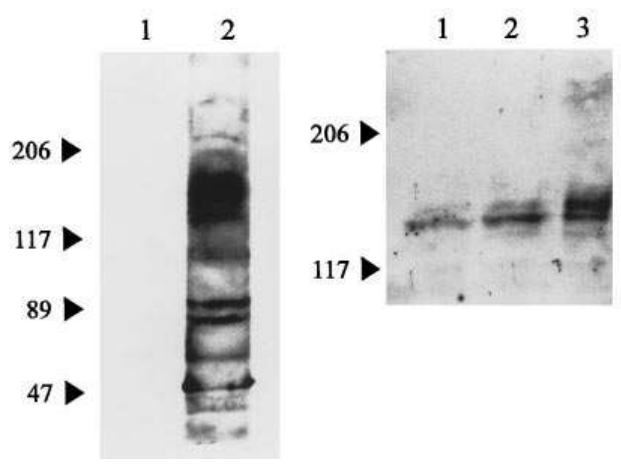

$\mathrm{C}$

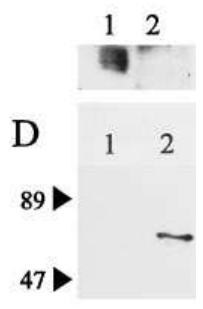

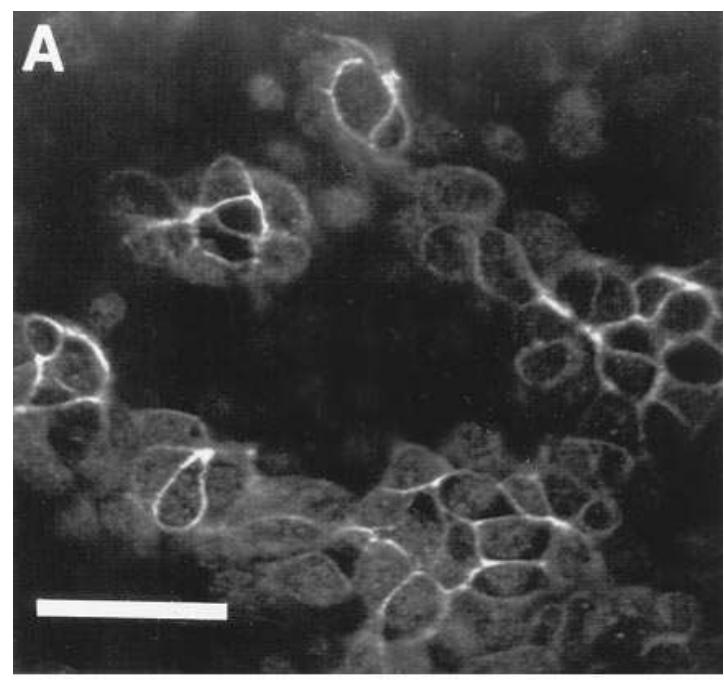
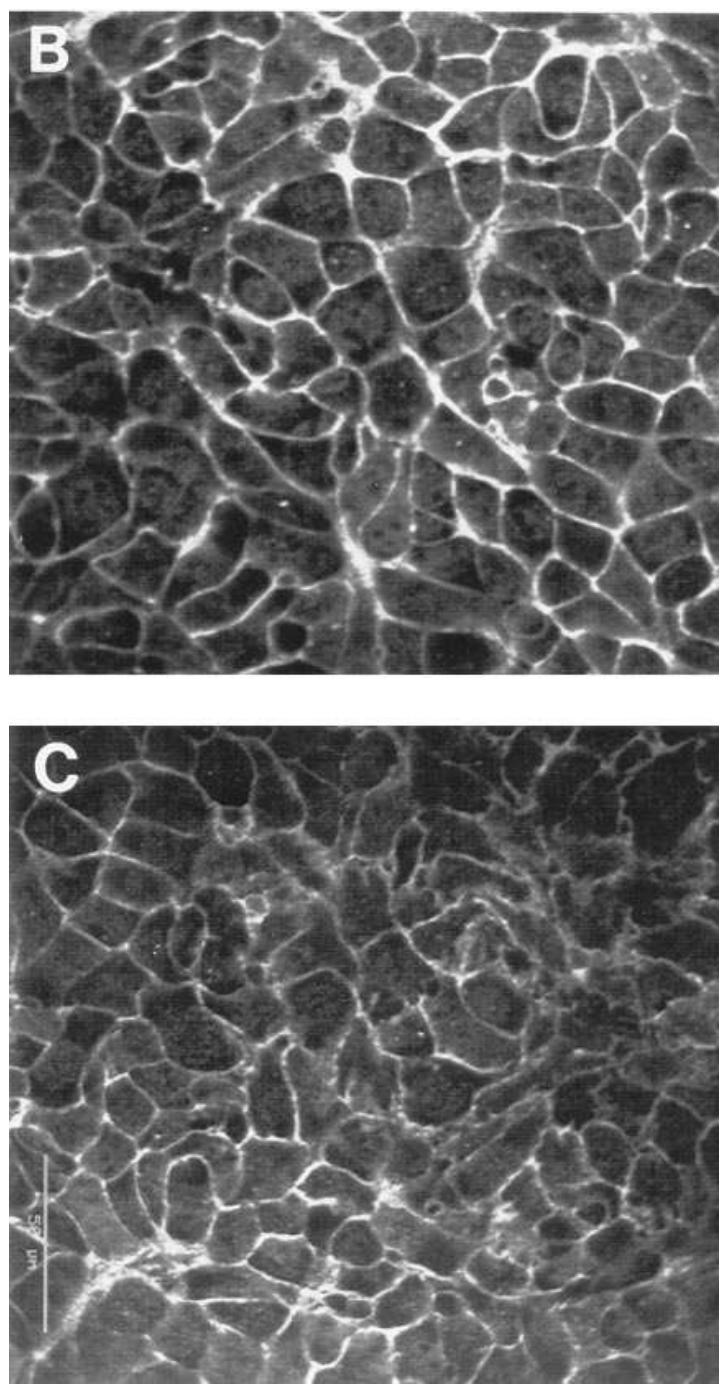

Figure 2. Immunolocalization of the $\mathrm{mBSC} 2$ protein in mIMCD-3 cells grown on polycarbonate filters. Confocal images were taken at three different levels of cell height: $(A)$ top, $(B)$ middle, and $(C)$ bottom. The immunostaining shows expression of the $\mathrm{mBSC} 2$ protein along the basolateral membrane. Bar, $50 \mu \mathrm{m}$.
Figure 1. Western blot of anti-mBSC2 on mouse kidney proteins. $(A)$ $8 \%$ SDS-PAGE showing a major broad band of $\sim 150 \mathrm{kD}$ and several smaller bands identified by anti-mBSC2 immune serum (lane 2), but not by preimmune serum (lane 1). (B) $6 \%$ SDS-PAGE using affinity-purified anti-mBSC2 on mouse cortical membranes $(400 \mu \mathrm{g})$ (lane 1), outer medulla membranes $(400 \mu \mathrm{g})$ (lane 2), and papillary membrane $(5-10 \mu \mathrm{g})$ (lane 3$)$ shows protein doublet of 140 and 150 $\mathrm{kD}$. (C) Broad 150-kD band identified by affinity-purified antimBSC2 (lane 1) is not seen when the affinity-purified anti-mBSC2 is preabsorbed with specific antigen (lane 2). (D) Affinity-purified anti$\mathrm{mBSC} 2$ recognizes the $\mathrm{mBSC} 2$ fusion protein (see Methods) (lane 2) but not an $\mathrm{rBSC} 1$ fusion protein made from the corresponding region of the protein (lane 1). 
Kidney sections. Male CD-1 mice (Charles River Laboratories) were perfusion fixed retrograde via the descending aorta with $4 \%$ paraformaldehyde in PBS. Kidneys were sliced and postfixed overnight in $4 \%$ paraformaldehyde at $4^{\circ} \mathrm{C}$. The tissue was then washed in PBS, cryoprotected in $30 \%$ sucrose overnight at $4^{\circ} \mathrm{C}$, imbedded in O.C.T. (Miles, Elkhart, IN), and finally frozen in isopentane cooled in dry ice. 5- $\mu \mathrm{m}$ frozen sections were cut with an RMC CMT-955A cryostat and thaw mounted on Superfrost Plus slides (Fisher Scientific, Pittsburgh, PA).

Indirect immunofluorescence. For immunofluorescence, tissue sections were incubated in $1 \%$ SDS and $8 \% 2$-mercaptoethanol for 5 min at room temperature followed by two 5-min washes in PBS. Sections were incubated for $30 \mathrm{~min}$ in PBS containing $1 \%$ BSA, and then incubated with affinity-purified anti-mBSC2 antibody diluted in PBS plus $1 \%$ BSA overnight at $4^{\circ} \mathrm{C}$. After washes in PBS, sections were incubated for $1 \mathrm{~h}$ with Cy3 conjugated anti-rabbit IgG (Jackson ImmunoResearch, West Grove, PA) at room temperature. For double labeling experiments, sections were incubated with anti-mBSC2 antibody and anti- $\alpha$ smooth muscle actin (clone 1A4; Sigma Immunochemicals, St. Louis, MO), or antirenin antibody (kindly provided by $\mathrm{T}$. Inagami [11]) and labeled with FITC-conjugated anti-mouse IgG (Jackson ImmunoResearch) or anti-rabbit IgG (Vector Labs, Burlingame, CA), respectively. For staining of macula densa, sections were incubated with anti-rBSC1 immune serum, an antibody specific for the apical Na-K-Cl cotransporter (6) and anti-Tamm-Horsfall an- tibody (antiuromucoid; Organon Teknika, Durham, NC) and labeled with FITC-conjugated anti-rabbit IgG (Vector Labs) and Texas redconjugated anti-goat IgG (Jackson ImmunoResearch), respectively. Sections were mounted with Vectashield (Vector Labs) and photographed with either a Nikon Microphot FXA microscope or a Bio Rad confocal microscope. Immunoabsorption of anti-mBSC2 was performed by incubating the purified antibody with specific antigen (the fusion protein) overnight at $4^{\circ} \mathrm{C}$ or with MBP as control.

For immunofluorescence staining of mIMCD3 cells, cells were grown to confluence on polycarbonate transwell filters (Costar Corp., Cambridge, MA) and processed as described above using affinitypurified anti-mBSC2 antibody.

\section{Results}

Western blots of mouse outer medulla membrane protein using anti-mBSC2 immune serum showed a major, broad band around $150 \mathrm{kD}$ (Fig. $1 A$, lane 2 ) which was not seen using preimmune serum (Fig. $1 A$, lane 1 ), as well as several bands of lower molecular mass. When the anti-mBSC2 immune serum was affinity purified using specific antigen, the broad band appeared to be composed of two distinct bands of 140 and $150 \mathrm{kD}$ which increased in intensity from cortex (Fig. $1 \mathrm{~B}$, lane 1 ) to outer
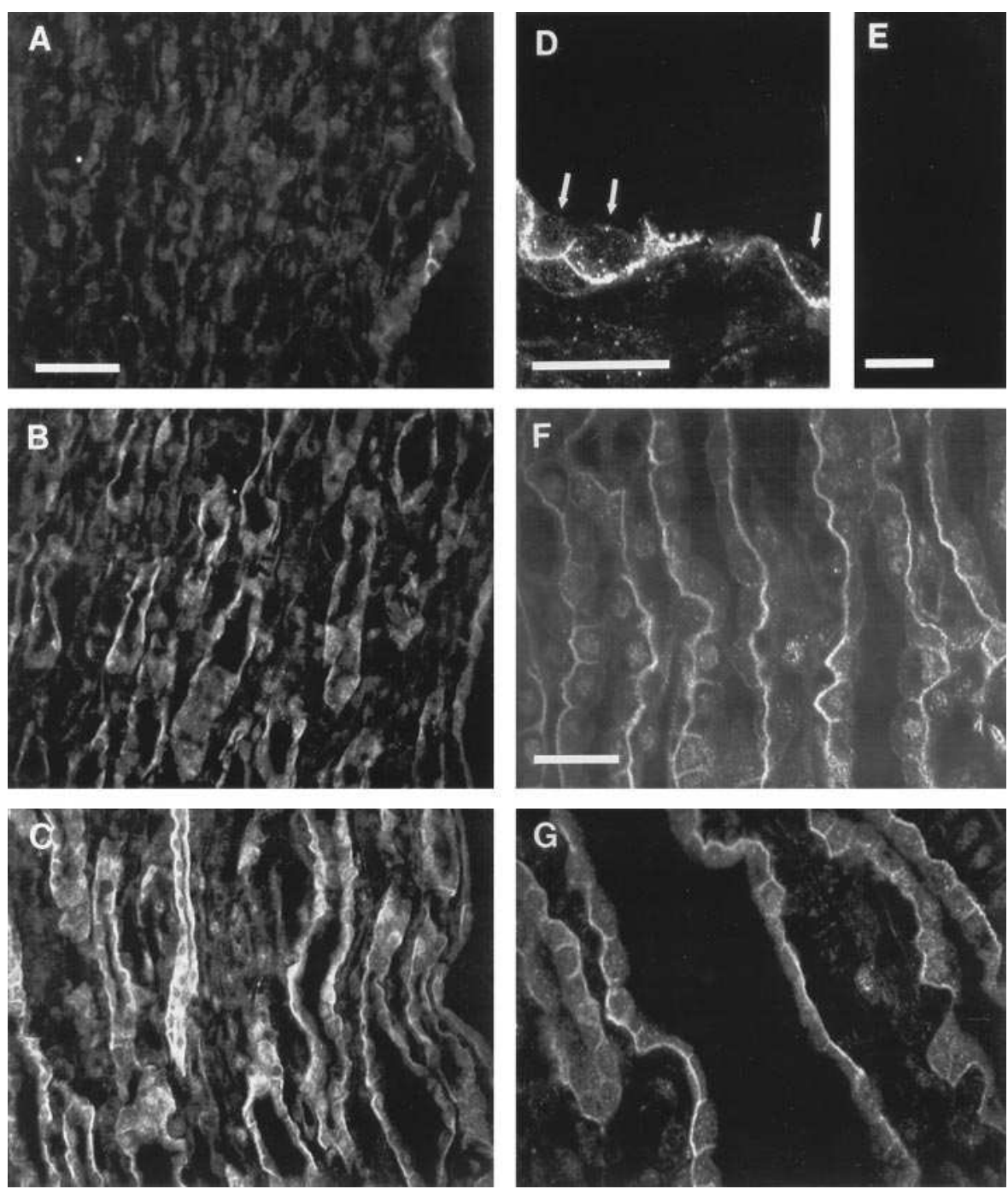

Figure 3. Confocal microscopy views showing the localization of the mBSC2 protein in the mouse inner medulla. IMCDs are stained with anti-mBSC2 antibody mostly in the terminal two-thirds of the IMCDs: a minimal signal is seen in the upper third $(A)$ but increases progressively from the middle $(B)$ to the terminal third $(C)$ of the inner medulla. High-power views of the collecting ducts in the terminal third of the inner medulla $(F$ and $G)$ show that the expression of the mBSC2 protein is along the basolateral, but not the apical membranes of the epithelial cells. The papillary surface epithelium seen on the right edge of the tissue sections $(A-C)$ shows equivalent intensity of staining from the upper to the terminal third of the inner medulla. As seen on the high-power view $(D)$, the basolateral membranes of the papillary surface epithelium are stained while the apical membranes, indicated by arrows, are negative. Light microscopy view of immunoabsorption of affinitypurified anti-mBSC2 $(E)$ shows an absence of signal in the terminal inner medulla. Bars, $A-C, 50 \mu \mathrm{m} ; D-G, 25 \mu \mathrm{m}$. 

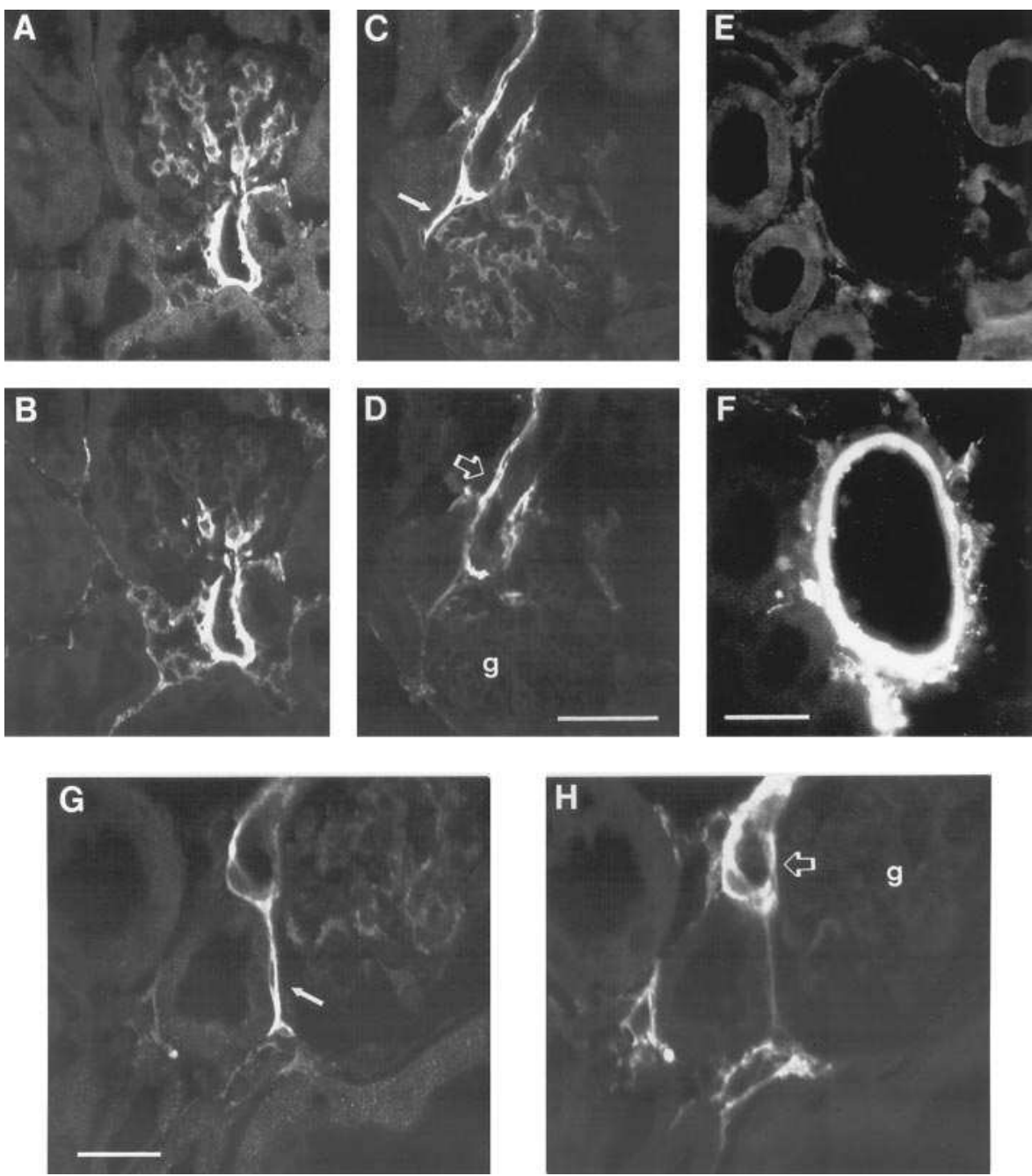

Figure 4. Renal colocalization of antimBSC2 with smooth muscle-specific anti$\alpha$-actin under confocal microscopy. A high level of expression of the $\mathrm{mBSC} 2$ protein $(A, C$, and $G)$ is seen in juxtaglomerular vasculature as identified by colocalization with anti- $\alpha$-actin $(B, D$, and $H$, open arrows). $\mathrm{mBSC} 2$ protein is also expressed in the glomerular mesangium $(A, C$, and $G)$ and in a neighboring juxtaglomerular structure which is negative for anti- $\alpha$-actin and probably represents extraglomerular mesangium ( $C$ and $G$, solid arrows).

Larger intrarenal arterioles were recognized by the smooth muscle-specific anti$\alpha$-actin $(F)$ but not by anti-mBSC2 $(E)$ antibodies. $g$, glomerulus. Bars, $A-D, 50 \mu \mathrm{m}$; $E-H, 25 \mu \mathrm{m}$. medulla (Fig. 1 B, lane 2) and were most intense in the inner medulla/papillary tissue (Fig. 1 B, lane 3 ). The lower molecular mass bands were not seen with this affinity-purified antibody. Note that the amount of inner medulla protein used on the Western blot in Fig. $1 \mathrm{~B}$ was $>40$-fold less than the amounts of cortex or outer medulla protein. The major signal around 150 $\mathrm{kD}$ was not seen when the affinity-purified anti-mBSC2 was preabsorbed with specific antigen (Fig. $1 C$ ), demonstrating the specificity of the $150-\mathrm{kD}$ signal for the mBSC2 protein. The specificity of anti-mBSC2 for BSC2 protein over BSC1 protein (the apical, absorptive $\mathrm{Na}-\mathrm{K}-\mathrm{Cl}$ cotransporter in the TAL) was demonstrated in Fig. $1 \mathrm{D}$ where anti-mBSC2 recognizes the mBSC2-MBP fusion protein (lane 2), but not an rBSC1-MBP fusion protein which consists of the comparable region of BSC1 (amino acids 834-900, lane 1). The 140-150-kD sizes seen on Western blot are consistent with the mBSC2 protein being a glycoprotein based on the $130-\mathrm{kD}$ size predicted by the coding region of the $m B S C 2$ gene. For comparison, human colonic $\mathrm{Na}-\mathrm{K}-\mathrm{Cl}$ cotransport protein (NKCC1) was found to be $170 \mathrm{kD}$ whereas the shark rectal gland $\mathrm{NKCC1}$ protein was $195 \mathrm{kD}$ with a core molecular mass of $135 \mathrm{kD}(9,12)$. Observation of a protein doublet suggests expression of different glycosylation or phosphorylation forms of the mBSC2 protein.

The immunopurified anti-mBSC2 was used to localize the mBSC2 protein in cells and tissue sections from mouse kidney.
Mouse mIMCD-3 cells, grown to confluence on filters, showed a clear basolateral pattern of staining with anti-mBSC2 (Fig. 2, $A-C)$, consistent with the putative basolateral location of mBSC2 suggested by ${ }^{86} \mathrm{Rb}$-uptake studies done in polarized mIMCD-3 cells (7).

Mouse kidney stained with affinity-purified anti-mBSC2 showed basolateral staining of the inner medulla/papilla, most intense in the terminal two-thirds of the collecting ducts (Fig. $3, A-C$ ) and along the papillary surface epithelium (Fig. $3 D$ ). Although the signal in IMCD is predominantly along the basolateral membrane (Fig. 3, $F$ and $G$ ), some intracellular signal is present which may represent background or localization of the cotransporter in intracellular compartments. No fluorescent signal was seen in outer medulla. The relatively light signal detected in outer medulla on Western blot with anti-mBSC2 (Fig. $1 B$ ) may represent a low level of protein expression not detectable by immunostaining of tissue sections or perhaps contamination from inner medulla on kidney dissection. Specificity of the antibody was demonstrated by absence of signal when anti-mBSC2 was preabsorbed with specific antigen (Fig. $3 E$ ) while no change in the signal was observed when the antibody was preabsorbed with the MBP (data not shown).

Close scrutiny of the renal cortex immunostained with antimBSC2 revealed an unexpected intense staining in the region of the juxtaglomerular apparatus and less intense staining 

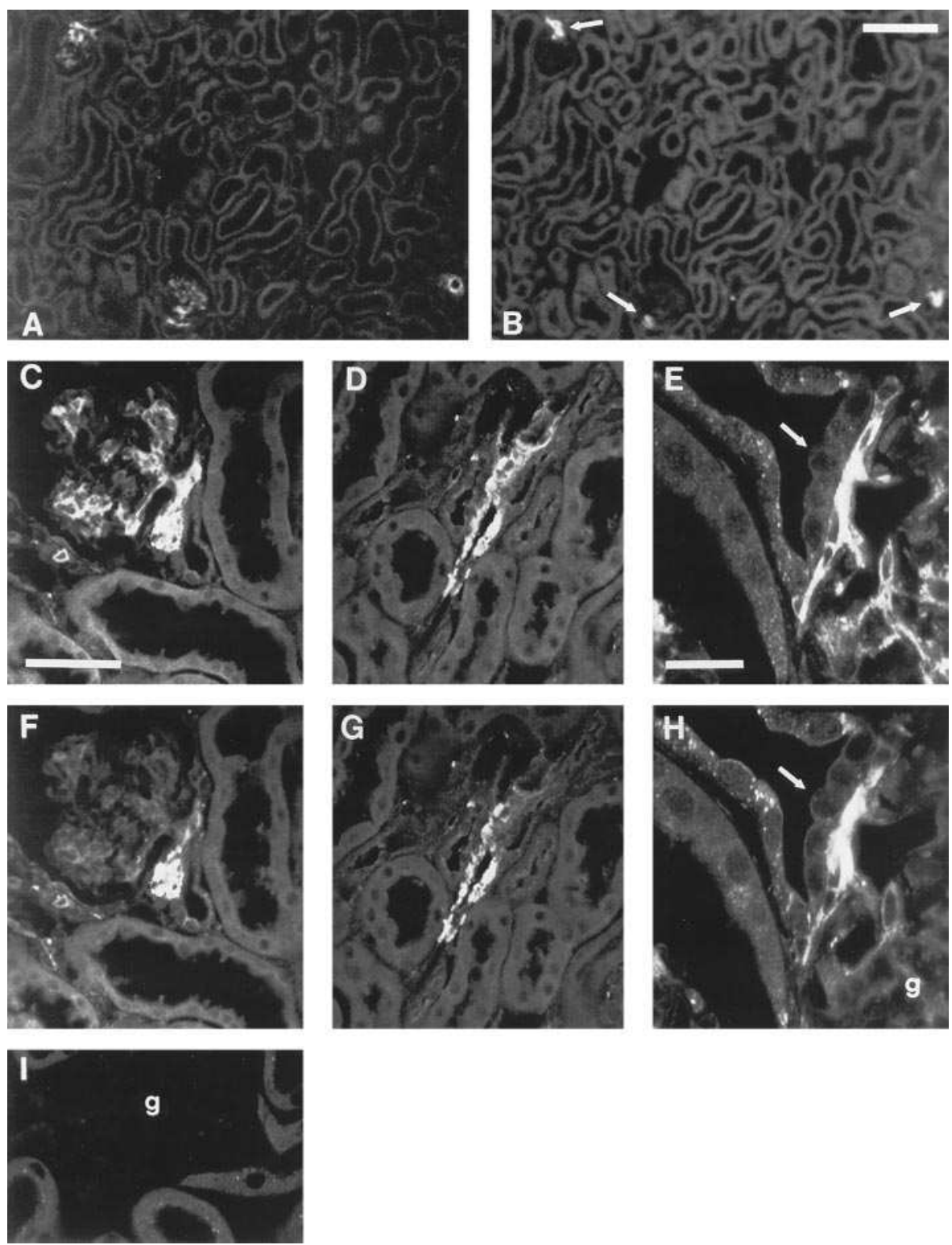

Figure 5. Renal colocalization of $\mathrm{mBSC} 2$ protein with renin demonstrates expression of mBSC2 in the granular cells of the afferent arteriole. All juxtaglomerular afferent arterioles labeled with the antirenin antibody ( $B$, solid arrows) also label with the anti-mBSC2 antibody $(A)$. Higher magnification demonstrates that the cells stained with antirenin $(F-H)$ also stain with anti-mBSC2 $(C-E)$ though the latter staining extends to cells which may be vascular smooth muscle cells or extraglomerular mesangial cells. The mBSC2 antibody also stains the glomerular mesangium. No mBSC2 signal is seen when the affinitypurified anti-mBSC2 antibody is preabsorbed with the mBSC2 fusion protein $(I)$. $g$, glomerulus; solid arrows, macula densa. Bars, $A$ and $B, 100 \mu \mathrm{m} ; C, D, F, G, I, 50$ $\mu \mathrm{m} ; E$ and $H, 25 \mu \mathrm{m}$. within the glomerular mesangium (Fig. 4, $A, C$, and $G$ ). No other cortical structures were recognized by the anti-mBSC2 antibody. To delineate the specific structures identified by the anti-mBSC2 antibody in the juxtaglomerular apparatus, colocalizations of anti-mBSC2 with smooth muscle-specific anti$\alpha$-actin antibody (Fig. 4) and antirenin antibody (Fig. 5) were performed. Fig. 4 shows expression of $\mathrm{mBSC} 2$ protein in juxtaglomerular vasculature which also labels with anti- $\alpha$-actin and in a juxtaglomerular structure which is negative for $\alpha$-actin and which morphologically appears to be extraglomerular mesangium. Anti-mBSC2 immunostaining was specific for the juxtaglomerular arteriole as other renal vasculature identified by anti- $\alpha$-actin staining (Fig. $4 F$ ) showed no signal when stained with anti-mBSC2 (Fig. $4 E$ ). Colocalization of antimBSC2 (Fig. 5, $A$ and $C-E$ ) with antirenin antibody (Fig. 5, $B$ and $F-H$ ) showed that the vasculature labeled by anti-mBSC2 was limited to the afferent arteriole. As indicated by the low magnification photographs (Fig. 5, $A$ and $B$ ) every glomerulus labeled with the antirenin antibody was positive with the antimBSC2 antibody. Upon close inspection, it appears that vascu- lar smooth muscle cells which stain positively for renin also exhibit mBSC2 protein, though there are some neighboring cells expressing the $\mathrm{mBSC} 2$ protein which do not stain for renin. Specificity of the antibody was again demonstrated by absence of signal when anti-mBSC2 was preabsorbed with specific antigen (Fig. $5 I$ ) while no change in the signal was observed when the antibody was preabsorbed with the MBP (data not shown).

Since macula densa cells are known to have apical bumetanide-inhibitable $\mathrm{Na}-\mathrm{K}-\mathrm{Cl}$ cotransport (13), we examined these cells for the presence of apical BSC1 and/or BSC2 isoforms of the $\mathrm{Na}-\mathrm{K}-\mathrm{Cl}$ cotransporter. The mouse macula densa, as identified by negative anti-Tamm-Horsfall immunostaining (14) in a juxtaglomerular CTAL (Fig. $6 \mathrm{~A}$ ), did not show expression of the mBSC2 protein (data not shown), but showed expression of the apical Na-K-Cl cotransporter, BSC1 (Fig. 6 $B)$, as reported previously in the rat (6). Confirming that antiBSC1 and anti-mBSC2 recognize distinct proteins in the mouse kidney, anti-BSC1 immunostains only the apical surfaces of MTALs and CTALs including the macula densa (6) whereas anti-mBSC2 immunostaining is specific for the affer- 
A

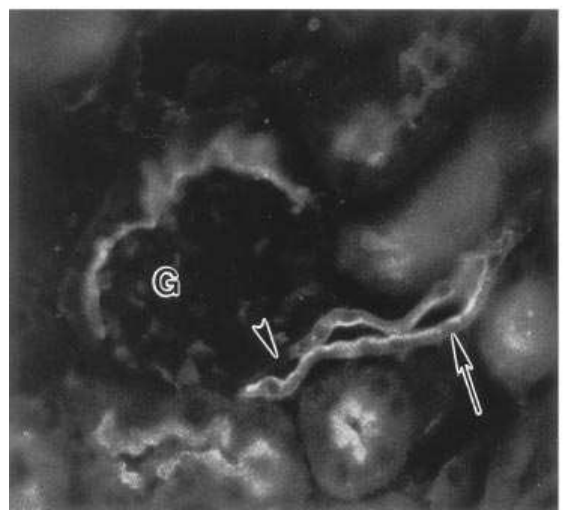

B

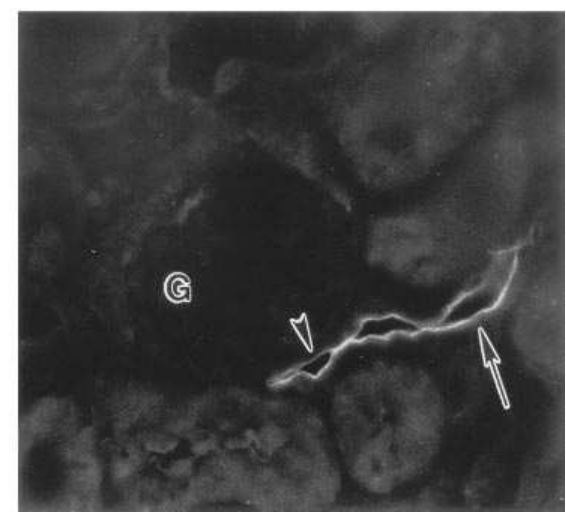

Figure 6. Mouse macula densa localization of $\mathrm{mBSC} 1$ protein. $(A)$ A macula densa region is identified by the absence of staining with anti-Tamm-Horsfall (arrowhead). $(B)$ The same macula densa cells exhibit presence of BSC1 (arrowhead) using antirBSC1 (6). Both anti-Tamm-Horsfall and anti-BSC1 labeled cortical thick limbs (arrows). $A$ and $B, \times 500$. ent arteriole, intra- and extraglomerular mesangium, and the terminal IMCD.

\section{Discussion}

The renal specific (BSC1 or NKCC2) and more widely expressed (BSC2 or NKCC1) isoforms of the Na-K-2Cl cotransporter are both highly expressed in the mammalian kidney (4, $5,7,15)$. In contrast to the BSC1 isoform, the precise localization of the $\mathrm{BSC} 2$ isoform within the kidney and its physiological function are unknown. In rabbit, the mRNA expression of the BSC2 isoform was shown to be low in cortex and outer medulla and to increase markedly from inner medulla to papilla (our unpublished observation), and in mouse, in situ hybridization showed high level of expression in glomeruli and distal nephron segments (16). This localization by Northern analysis and in situ hybridization is compatible with the high level of expression found in the mouse inner medullary collecting duct cell line, mIMCD-3, which was used to isolate the cDNA encoding the mBSC2 cotransporter. The aim of this study was to localize this putative basolateral isoform of the $\mathrm{Na}-\mathrm{K}-2 \mathrm{Cl}$ cotransporter in the mouse kidney. For this purpose, we generated a polyclonal antibody which, as shown in Fig. $1, A-C$, specifically recognizes a protein doublet of $\sim 140$ and $150 \mathrm{kD}$. Anti-mBSC2 antibody was shown to be specific for the basolateral isoform as demonstrated by the absence of reactivity against the BSC1 fusion protein (Fig. $1 \mathrm{D}$, the two fusion proteins being designed in corresponding regions of the respective cotransporter proteins) and by the absence of any signal in MTAL or CTAL where BSC1 is highly expressed on apical membranes (6).

$\mathrm{mBSC} 2$ protein localized in the kidney to two distinct regions: the papillary IMCD, as anticipated based on the origin of the mBSC 2 cDNA clone from the mouse mIMCD-3 cell line, and unexpectedly, the glomerulus and the juxtaglomerular apparatus. In the mouse papilla, $\mathrm{mBSC} 2$ protein was located mainly in the terminal two-thirds of the IMCD, in a contiguous pattern consistent with previous reports of a single cell type in this region (17). Although some intracellular staining was observed, the mBSC2 protein localized predominantly to the basolateral surface of the IMCD cells. Expression of an $\mathrm{Na}-\mathrm{K}-2 \mathrm{Cl}$ cotransporter in the IMCD is consistent with previous reports which showed bumetanide-sensitive ${ }^{86} \mathrm{Rb}$ uptake in rat and mouse IMCD cells with functional basolateral location of the Na-K-2Cl cotransporter demonstrated in the mouse IMCD cells $(7,18)$. Since the papillary osmolarity can reach very high levels and is subject to great variability, the volume regulatory function of the basolateral $\mathrm{Na}-\mathrm{K}-2 \mathrm{Cl}$ cotransporter (19) may be particularly important in this segment. Although the IMCD segment is generally believed to mediate $\mathrm{Na}^{+}$reabsorption through apical $\mathrm{Na}^{+}$channels and basolateral $\mathrm{Na}^{+} / \mathrm{K}^{+}$ pumps, it has also been reported that atrial natriuretic peptide stimulates $\mathrm{NaCl}$ secretion via a furosemide-inhibitable $\mathrm{Na}-\mathrm{K}$ $2 \mathrm{Cl}$ cotransporter (20). These results are consistent with the upregulation in mIMCD-3 cells of Na-K-2Cl cotransporter expression by atrial natriuretic peptide (21), a hormone otherwise known to downregulate the expression of the $\mathrm{Na}^{+}$channel and the $\mathrm{Na}^{+} / \mathrm{K}^{+}$pump $(22,23)$. The papillary interstitium is also known to contain high amounts of ammonium. Since both $\mathrm{NH}_{4}{ }^{+}$and $\mathrm{H}^{+}$secretion have been demonstrated in isolated rat IMCD (24), and since the $\mathrm{NH}_{4}^{+}$cation can compete with the $\mathrm{K}^{+}$binding site on the cotransporter (25), the basolateral Na$\mathrm{K}-2 \mathrm{Cl}$ cotransporter in the IMCD could constitute one of the major routes for $\mathrm{H}^{+}$and $\mathrm{NH}_{4}^{+}$secretion. This is of particular importance in light of recent findings demonstrating a primary role for the basolateral $\mathrm{Na}-\mathrm{K}-2 \mathrm{Cl}$ cotransporter in acid secretion in the stomach (26).

Other structures within the papilla (i.e., thin limbs) exhibited only very faint signal with anti-mBSC2 which may either represent very low levels of expression or background staining. The signal seen on the basolateral surface of the terminal IMCD cells becomes fainter in the more proximal IMCD and disappears before reaching the outer medulla. No signal was observed in outer medulla. A strong signal was also seen on the basolateral aspect of the papillary surface epithelium which is in contrast to a previous functional study of rabbit papillary epithelium showing apical Na-K-Cl cotransport (27).

In the cortex, anti-mBSC2 intensely labeled the smooth muscle cells of the juxtaglomerular afferent arteriole as demonstrated by colocalization with antirenin and anti- $\alpha$-actin antibodies. No other vasculature was stained for mBSC2. Immunostaining was also observed within the glomerular mesangium (Fig. 4, $A$ and $C$, and Fig. 5, $C$ and $E$ ) where the cotransporter has been identified previously by both transport and $\left[{ }^{3} \mathrm{H}\right]$ bumetanide-binding experiments (28). A strong signal was seen in a juxtaglomerular structure which was negative for anti- $\alpha$-actin and which most likely represents extraglomerular mesangium (Fig. 4, $C, D, G$, and $H$ ). While all cells recognized 


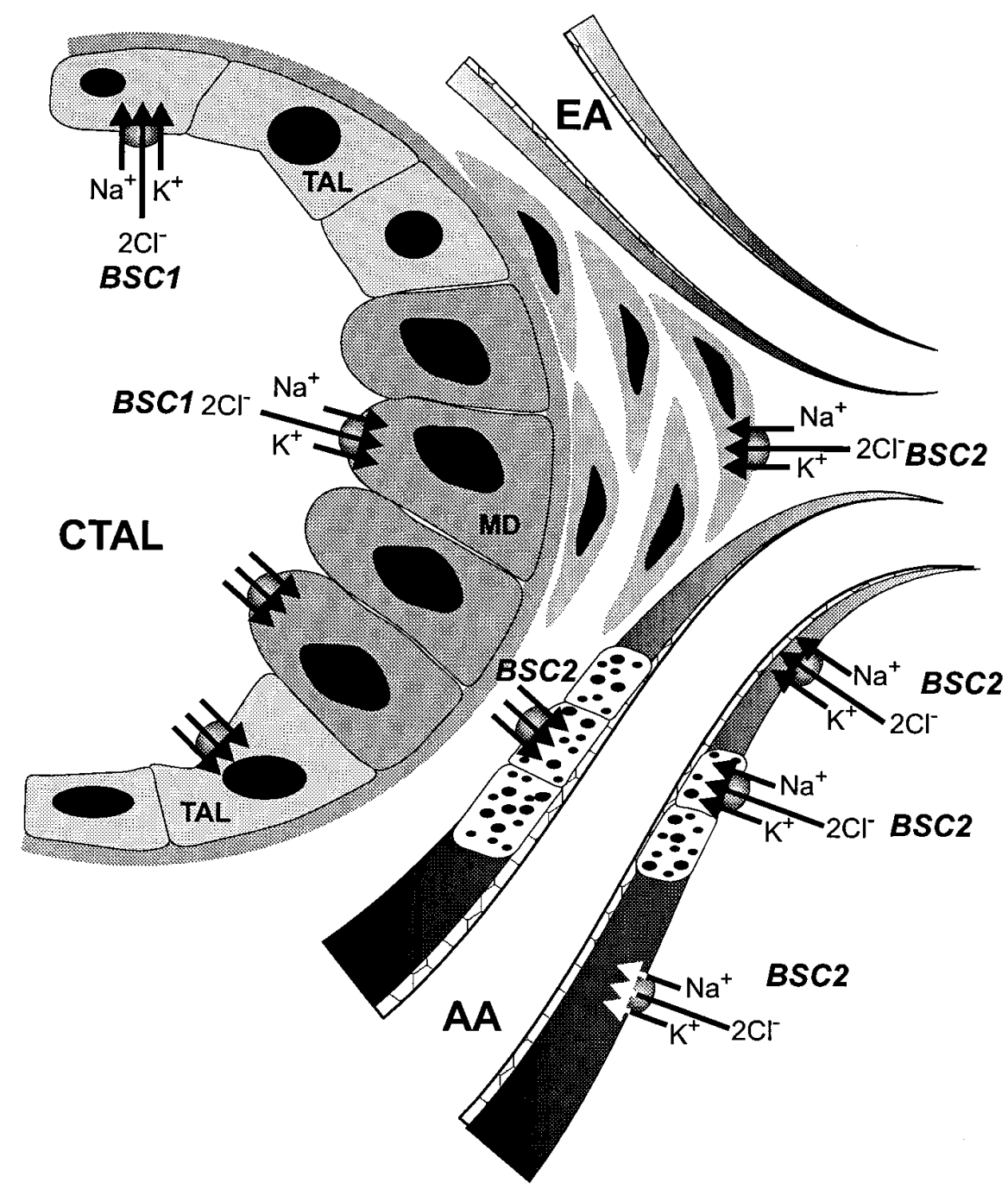

Figure 7. Diagram showing the localization of the two isoforms of the Na-K-2Cl cotransporter BSC1 and BSC2 in the juxtaglomerular vasculature and the macula densa. $M D$, macula densa; $C T A L$, cortical thick ascending limb; $A A$, afferent arteriole; $E A$, efferent arteriole. Dotted cells represent granular cells.

by antirenin were also recognized by anti-mBSC2, mBSC 2 expression was also seen in some neighboring smooth muscle cells which did not stain for renin. This specific localization of the cotransporter to the extraglomerular mesangium and to a subpopulation of smooth muscle cells of the afferent arteriole suggests a role for the mBSC2 protein in tubuloglomerular feedback (TGF) and/or renin secretion.

TGF is a mechanism by which an increased delivery of $\mathrm{NaCl}$ in the CTAL affects the tone of the afferent arteriole at the entrance to the glomerulus and consequently alters the rate of filtration in the glomerulus (29). This increased delivery of $\mathrm{NaCl}$ is thought to be sensed in the CTAL by macula densa cells by means of a furosemide-inhibitable Na-K-2Cl cotransporter which appears to be the apical renal-specific isoform of the $\mathrm{Na}-\mathrm{K}-2 \mathrm{Cl}$ cotransporter, BSC1, as seen in Fig. 6. The mechanism of transmission of signal between the macula densa and the afferent arteriole is the subject of much speculation, but it is known that increased $\mathrm{Cl}^{-}$delivery to the macula densa results in $\mathrm{Cl}^{-}$accumulation in the juxtaglomerular interstitium. The presence of the basolateral isoform of the Na-K$2 \mathrm{Cl}$ cotransporter on extraglomerular mesangial cells and smooth muscle cells of the juxtaglomerular afferent arteriole (Figs. 4 and 5) would be consistent with a direct role for $\mathrm{Cl}^{-}$in transmitting the TGF signal from the macula densa to the afferent arteriole.

Additionally, this $\mathrm{Cl}^{-}$sensing pathway from $\mathrm{BSC} 1$ in the macula densa to BSC2 in the extraglomerular mesangium and the afferent arteriole may be important in renin secretion which is activated by a decrease in $\left[\mathrm{Cl}^{-}\right]$in the CTAL (30). Both TGF and renin release are known to be inhibited by furosemide (31-33). Although this inhibitory effect of furosemide may take place through the macula densa apical Na-K$2 \mathrm{Cl}$ cotransporter (BSC1), it is also possible that TGF and renin release are inhibited by furosemide acting on the BSC2 isoform in the extraglomerular mesangium and/or the afferent arteriole. This has potentially important clinical implications in that a drug which would preferentially inhibit BSC2 in the juxtaglomerular region and not BSC1 in the macula densa might be useful in high renin hypertension and some other high renin states.

In summary, we have developed an antibody against BSC2, the widely expressed isoform of the $\mathrm{Na}-\mathrm{K}-2 \mathrm{Cl}$ cotransporter, and studied its localization in the kidney. The BSC2 protein is expressed on the basolateral surface of the papillary surface epithelium and on the basolateral surface of the terminal IMCD cells where it may play a significant role in salt and/or 
acid secretion, and unexpectedly in glomerular mesangium, juxtaglomerular cells which most probably represent extraglomerular mesangium, and in vascular smooth muscle cells of the afferent arteriole including the renin-secreting granular cells (Fig. 7). The BSC2 cotransporter may be part of the signaling mechanism between the macula densa and the afferent arteriole, an hypothesis which needs further study.

\section{Acknowledgments}

This work was supported in part by National Institutes of Health (NIH) NRSA grants to M.R. Kaplan (DK-08898) and M.D. Plotkin (DK-08887); by NIH grants DK-42956 to D. Brown and DK-38603 to S.C. Hebert; and by grants from NIH (HL-49251) and from the American Heart Association, Massachusetts Affiliate (Grant-in-Aid 13-592-912) to E. Delpire.

\section{References}

1. Hebert, S.C., and S.R. Gullans. 1995. The electroneutral sodium-(potassium)-chloride co-transporter family: a journey from fish to the renal co-transporters. Curr. Opin. Nephrol. Hypert. 4:389-391.

2. Kaplan, M.R., D.B. Mount, E. Delpire, G. Gamba, and S.C. Hebert. 1996. Molecular mechanisms of $\mathrm{NaCl}$ cotransport. Annu. Rev. Physiol. 58:649668.

3. Quaggin, S.E., J.A. Payne, B. Forbush III, and P. Igarashi. 1995. Localization of the renal Na-K-Cl cotransporter gene (Slc12aI) on mouse chromosome 2. Mamm. Genome. 6:557-561.

4. Gamba, G., A. Miyanoshita, M. Lombardi, J. Lytton, W.-S. Lee, M. Hediger, and S.C. Hebert. 1994. Molecular cloning, primary structure, and characterization of two members of the mammalian electroneutral sodium-(potassium)-chloride cotransporter family expressed in kidney. J. Biol. Chem. 269: 17713-17722.

5. Igarashi, P., G.B. Vanden Heuvel, J. Payne, and B.I. Forbush. 1995. Cloning, embryonic expression and alternative splicing of a murine kidney specific Na-K-Cl cotransporter. Am. J. Physiol. (Renal Fluid Electrolyte Physiol.) 269:F405-F418.

6. Kaplan, M.R., M.D. Plotkin, W.-S. Lee, Z.-C. Xu, J. Lytton, and S.C. Hebert. 1996. Apical localization of the Na-K-2Cl cotransporter, rBSC1, on rat thick ascending limbs. Kidney Int. 49:40-47.

7. Delpire, E., M.I. Rauchman, D.R. Beier, S.C. Hebert, and S.R. Gullans. 1994. Molecular cloning and chromosome localization of a putative basolateral $\mathrm{Na}-\mathrm{K}-2 \mathrm{Cl}$ cotransporter from mouse inner medullary collecting duct (mIMCD3) cells. J. Biol. Chem. 269:25677-25683.

8. Xu, J.-C., C. Lytle, T.T. Zhu, J.A. Payne, E.J. Benz, and B. Forbush III. 1994. Molecular cloning and functional expression of the bumetanide-sensitive Na-K-2Cl cotransporter. Proc. Natl. Acad. Sci. USA. 91:2201-2205.

9. Payne, J.A., J.-C. Xu, M. Hass, C.Y. Lytle, D. Ward, and B. Forbush III. 1995. Primary structure, functional expression, and chromosome localization of the bumetanide sensitive Na-K-Cl cotransporter in human colon. J. Biol. Chem. 270:17977-17985.

10. Cook, D.I., and J.A. Young. 1993. Epithelial secretion driven by anions other than chloride. News Physiol. Sci. 8:91-93.

11. Celio, M.R., and T. Inagami. 1981. Angiotensin II immunoreactivity coexists with renin in the juxtaglomerular granular cells of the kidney. Proc. Natl. Acad. Sci. USA. 78:3897-3900.

12. Lytle, C., J.-C. Xu, D. Biemesderfer, M. Haas, and B. Forbush III. 1992. The Na-K-Cl cotransport protein of shark rectal gland. I. Development of monoclonal antibodies, immunoaffinity purification, and partial biochemical charac- terization. J. Biol. Chem. 267:25428-25437.

13. Lapointe, J.-Y., D. Bell, and J. Cardinal. 1990. Direct evidence for apical $\mathrm{Na}^{+}: 2 \mathrm{Cl}^{-}: \mathrm{K}^{+}$cotransport in macula densa cells. Am. J. Physiol. (Renal Fluid Electrolyte Physiol.) 258:F1466-F1469.

14. Hoyer, J.R., S.P. Sisson, and R.L. Vernier. 1979. Tamm-Horsfall glycoprotein. Ultrastructural immunoperoxidase localization in rat kidney. Lab. Invest. 41:168-173.

15. Payne, J.A., and B. Forbush III. 1994. Alternatively spliced isoforms of the putative renal Na-K-Cl cotransporter are differentially distributed within the rabbit kidney. Proc. Natl. Acad. Sci. USA. 91:4544-4548.

16. Vanden Heuvel, G.B., J.A. Payne, B. Forbush III, and P. Igarashi. 1995. Expression of the basolateral Na-K-Cl cotransporter (NKCC1) during murine nephrogenesis and embryonic development. J. Am. Soc. Nephrol. 6:354 (Abstr).

17. Masden, K.M., W.L. Clapp, and J.W. Verlander. 1988. Structure and function of the inner medullary collecting duct. Kidney Int. 34:441-454.

18. Grupp, C., I. Pavenstadt-Grupp, R.W. Grunewald, C. Bevan, J.B. Stokes, and R.K. Kinne. 1989. A Na-K-2Cl cotransporter in isolated rat papillary collecting duct cells. Kidney Int. 36:201-209.

19. Palfrey, H.C., and M. O'Donnell. 1992. Characteristics and regulation of the $\mathrm{Na} / \mathrm{K} / 2 \mathrm{Cl}$ cotransporter. Cell. Physiol. Biochem. 2:293-307.

20. Rocha, A.S., and L.H. Kudo. 1990. Atrial peptide and cGMP effects on $\mathrm{NaCl}$ transport in inner medullary collecting duct. Am. J. Physiol. (Renal Fluid Electrolyte Physiol.) 259:F258-F268.

21. Delpire, E., M.I. Rauchman, S.C. Hebert, and S.R. Gullans. 1993. Partial cDNA cloning and hormonal regulation of a basolateral Na-K-2Cl cotransporter from the mouse terminal inner medullary collecting duct (IMCD). $J$. Gen. Physiol. 102:18a. (Abstr.)

22. Zeidel, M.L., D. Kikeri, P. Silva, M. Burrowes, and B.M. Brenner. 1988. Atrial natriuretic peptides inhibit conductive sodium uptake by rabbit inner medullary collecting duct cells. J. Clin. Invest. 82:1067-1074.

23. Sonnenberg, H., U. Honrath, and D.R. Wilson. 1990. In vivo microperfusion of inner medullary collecting duct in rats: effect of amiloride and ANF. Am. J. Physiol. (Renal Fluid Electrolyte Physiol.) 259:F222-F226.

24. Wall, S.M., J.M. Sands, M.F. Flessner, H. Monogushi, K.R. Spring, and M.A. Knepper. 1990. Net acid transport by isolated perfused inner medullary collecting ducts. Am. J. Physiol. (Renal Fluid Electrolyte Physiol.) 258:F65-F84.

25. Kinne, R., B. Koenig, J. Hannafin, E. Kimme-Saffram, D.M. Scott, and K. Zierold. 1985. The use of membrane vesicles to study the $\mathrm{NaCl} / \mathrm{KCl}$ cotransporter involved in active transepithelial chloride transport. Pflügers Arch. 405: S101-S105.

26. Soybel, D.I., S.R. Gullans, F. Maxwell, and E. Delpire. 1995. Role of basolateral Na-K-Cl cotransport in $\mathrm{HCl}$ secretion by amphibian gastric mucosa. Am. J. Physiol. (Cell Physiol.) 269:C242-C249.

27. Sands, J.M., M.A. Knepper, and K.R. Spring. 1986. Na-K-Cl cotransport in apical membrane of rabbit renal papillary surface epithelium. Am. J. Physiol. (Renal Fluid Electrolyte Physiol.) 251:F475-F484.

28. Homma, T., and R.C. Harris. 1991. Time-dependent biphasic regulation of $\mathrm{Na}+/ \mathrm{K}+/ \mathrm{Cl}-$ cotransport in rat glomerular mesangial cells. J. Biol. Chem. 266:13553-13559.

29. Schnermann, J., F.S. Wright, J.M. Davis, W. von Stackelberg, and G. Grill. 1970. Regulation of superficial nephron filtration rate by tubulo-glomerular feedback. Pflügers Arch. 318:147-175.

30. Kirchner, K.A., T.A. Kotchen, J.H. Galla, and R.G. Luke. 1978. Importance of chloride for acute inhibition of renin by sodium chloride. Am. J. Physiol. (Renal Fluid Electrolyte Physiol.) 235:F444-F450.

31. Ito, S., and O. Carretero. 1990. An in vitro approach to the study of macula densa-mediated glomerular hemodynamics. Kidney Int. 328:1206-1210.

32. Lorenz, J.N., H. Weihprecht, J. Schnermann, O. Skøtt, and J.P. Briggs. 1991. Renin release from isolated juxtaglomerular apparatus depends on macula densa chloride transport. Am. J. Physiol. (Renal Fluid Electrolyte Physiol.) 260:F486-F493.

33. He, X.-R., S.G. Greenberg, J.P. Briggs, and J. Schermann. 1995. Effect of furosemide and verapamil on the $\mathrm{NaCl}$ dependency of macula densa-mediated renin secretion. Hypertension (Dallas). 26:137-142. 